http://jmscr.igmpublication.org/home/ ISSN (e)-2347-176x ISSN (p) 2455-0450 crossref DOI: https://dx.doi.org/10.18535/jmscr/v7i8.90

\title{
Impaired Glucose Regulation in Cirrhosis Liver - The Utility of Oral Glucose Tolerance Test
}

\author{
Authors \\ Dr Kiruthika Kesavan $\mathrm{MD}^{1}$, Dr P. Sureshkumar $\mathrm{MD}^{2^{*}}$ \\ ${ }^{1}$ Assistant Professor, Dept. of General Medicine, Govt. Sivagangai Medical College \\ ${ }^{2}$ Assistant Professor, Dept. of General Medicine, Govt. Sivagangai Medical College \\ *Corresponding Author \\ Dr P. Sureshkumar MD. \\ Assistant Professor, Dept. of General Medicine, Govt. Sivagangai Medical College
}

\begin{abstract}
Background and Objectives: Liver is one of the major organs to be involved in carbohydrate metabolism, and hence it is no surprise that chronic liver disease is associated with derangements in glucose metabolism. The objectives of this study is to find out the prevalence and clinical implications of the spectrum of glucose metabolism disorders (IGT/DM) in CLD and assess and compare the prognosis of the disease using the Child-Turcotte-Pugh scoring.

Methods: This study was conducted over a period of six months and included 50 patients. Detailed clinical examination along with biochemical and radiological investigations were carried out. OGTT was then performed in these individuals. Patients who were known diabetics, drugs causing hyperglycemia and pregnant women were excluded from the study.

Results: CLD was much more common in males. There was a significant percentage of IGT/DM diagnosed in these patients using OGTT. The results were, 36\%-IGT and 28\% DM. It was also proved that as the severity of disease worsened, which was assessed by CTP scoring, the degree of hyperglycemia also increase proportionately. The percentage of impaired glucose regulation in Child class A was $47 \%$, class B $68 \%$ and class C 93\%
\end{abstract}

Keywords: Chronic liver disease, oral glucose tolerance test, impaired glucose tolerance, diabetes mellitus.

\section{Introduction}

Liver plays a central role in blood glucose regulation $^{1}$ and it is proved beyond doubt that chronic liver disease is associated with abnormalities of metabolism of carbohydrates. Advanced liver diseases manifest with metabolic derangements and this is regardless of the aetiology of the disease. To some extent, the abnormalities observed in the late stages of cirrhosis, regardless of the cause, are similar ${ }^{2}$. The liver is responsible for the maintenance of the blood sugar levels within normal limits by various mechanisms such as gluconeogenesis, glycogenesis and glycogenolysis. The blood glucose levels are very low in fulminant acute hepatitis; however this is in contrast with chronic liver disease where the incidence of diabetes and impaired glucose tolerance are of a significant percentage. The contribution to energy in fasting state is more by fats than the carbohydrates ${ }^{3}$.

The incidence of diabetes is increased in patients with chronic hepatitis $\mathrm{C}^{4,5}$, other metabolic 
disorders like hemochromatosis and autoimmune hepatitis.

The degree of liver dysfunction ${ }^{6}$ directly correlates with the extent of carbohydrate metabolism disturbances. The metabolic changes in chronic liver diseases are complex and not fully understood $^{6,7}$.

OGTT remains superior ${ }^{8}$ to the same test carried out through the intravenous route till date, because this gives an opportunity for the stimulation of secretion of insulin and other hormones normally.

\section{Aims and Objectives}

1. To study the various clinical, biochemical and radiological manifestations of Chronic Liver Disease.

2. To determine the prevalence and clinical implications of the spectrum of glucose metabolism disorders (Impaired Glucose Tolerance/ Diabetes Mellitus) in patients with chronic liver disease.

3. To correlate the glycemic levels with the class of Child Turcotte Pugh Scoring System, in assessing the prognosis of the disease.

\section{Materials and Methods}

This is an observational study conducted in Govt sivagangai Medical College, sivagangaai over a period of six months duration.

The study population included 50 patients who attended the Department of Medicine, both as outpatient or inpatient, and were diagnosed to have chronic liver disease. The individuals were taken up for the study after getting their informed consent.

Study Design: Cross sectional study

Period of Study: Six Months (Feb 2019 - July 2019)

\section{Ethical Committee Approval: Approved}

This study was approved by the Ethical Committee, Sivagangai Medical College.

\section{Statistical Analysis:}

The significance testing was done using the Chi Square Test. The results were considered significant if the 'p' value was below 0.05 .

\section{Observations \& Results}

During the 6 month study period, a total number of 50 patients were studied for finding out the prevalence of impaired glucose tolerance and diabetes mellitus in patients with cirrhosis liver, and its relation to other selected variables.

\section{Age Distribution}

Chart No 1 - Age Distribution among the population1

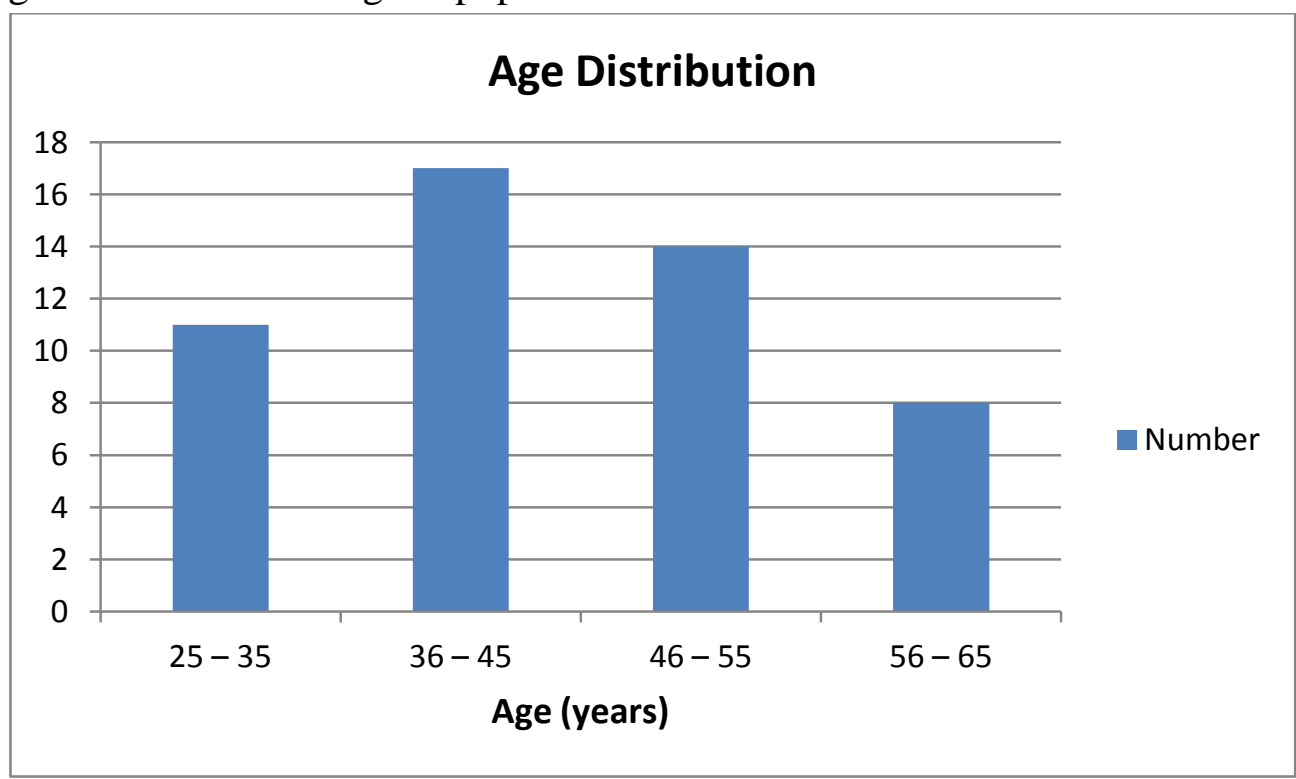


The age of the patients range from 29 to 65 years. The majority of the patients were within the age group of 36 to 45 years (34\%). This was followed closely by patients within the age group of 46 to 55 years $(28 \%)$. The mean age group was 45.42 years.

\section{Sex Distribution}

Chart No 2 - Sex Distribution among the study population

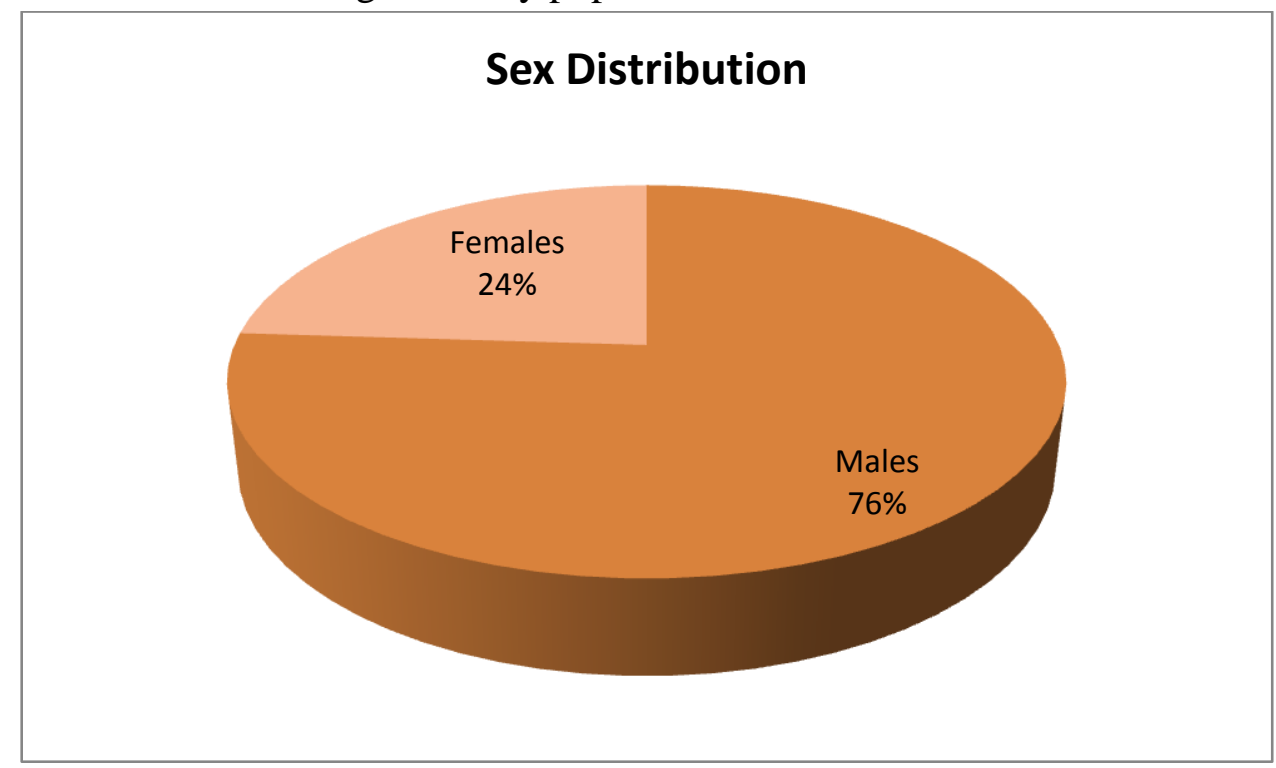

Child Turcotte Pugh Classification

Chart No 3 - Distribution of cases within the CTP Class

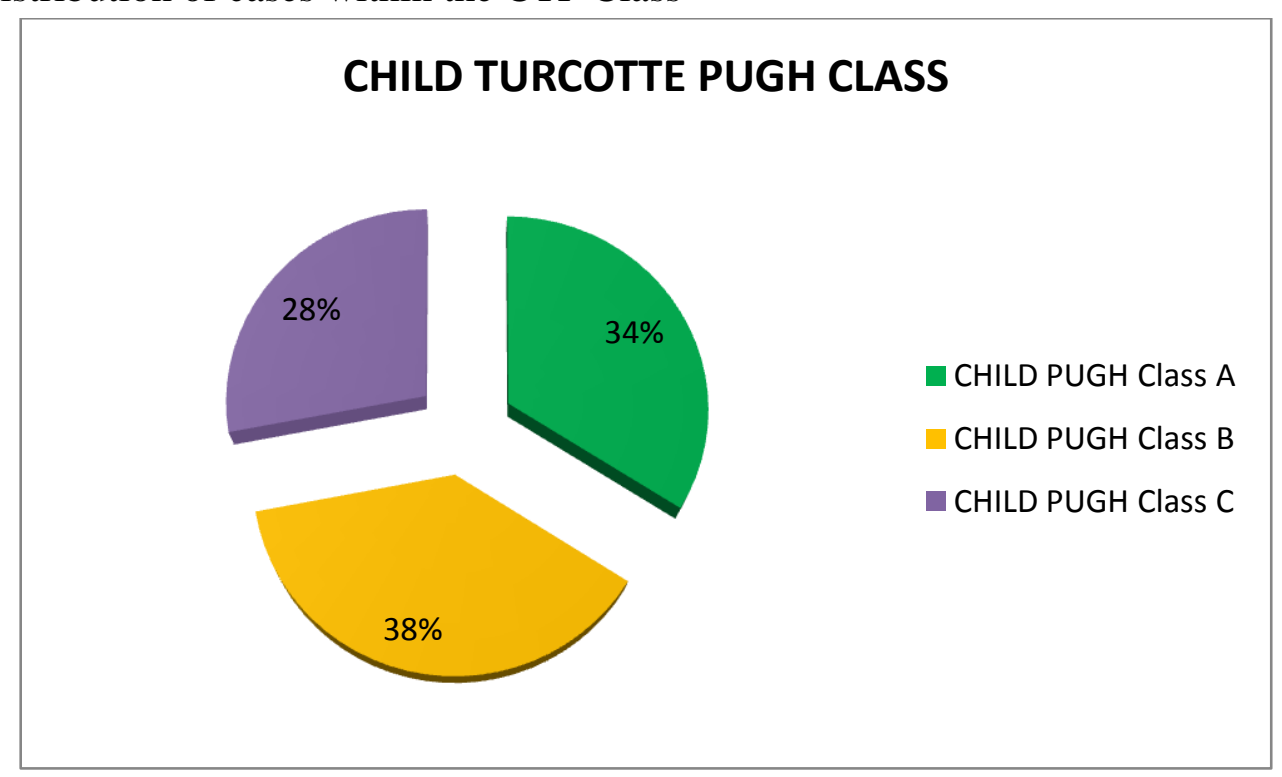

Of the 50 patients under this study, the number of cases in the various classes of the CTP classification were as follows - Class A: 17 (34\%), Class B: 19 (38\%), Class C: 14 (28\%). The maximum number of patients were within the class B.

\section{Oral Glucose Tolerance Test}

50 patients in this were subjected to the oral glucose tolerance test, by administration of $75 \mathrm{~g}$ of glucose in $300 \mathrm{ml}$ of water and measuring the plasma glucose levels at $0,1 / 2,1,1 \frac{1}{2}$ and $2 \mathrm{hrs}$. The results of the test are as follows. 
Chart No 4 - Results of OGTT

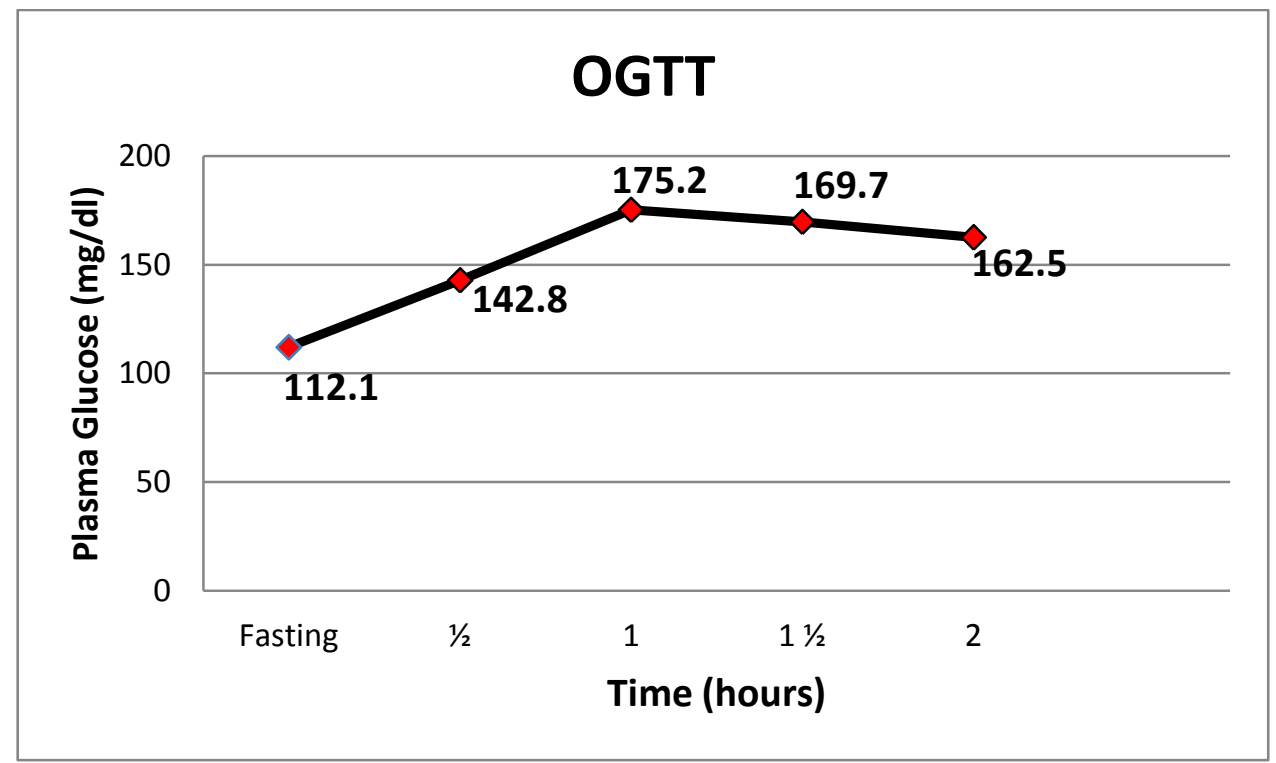

Chart No 5 - Diagram showing Distribution of Results of OGTT

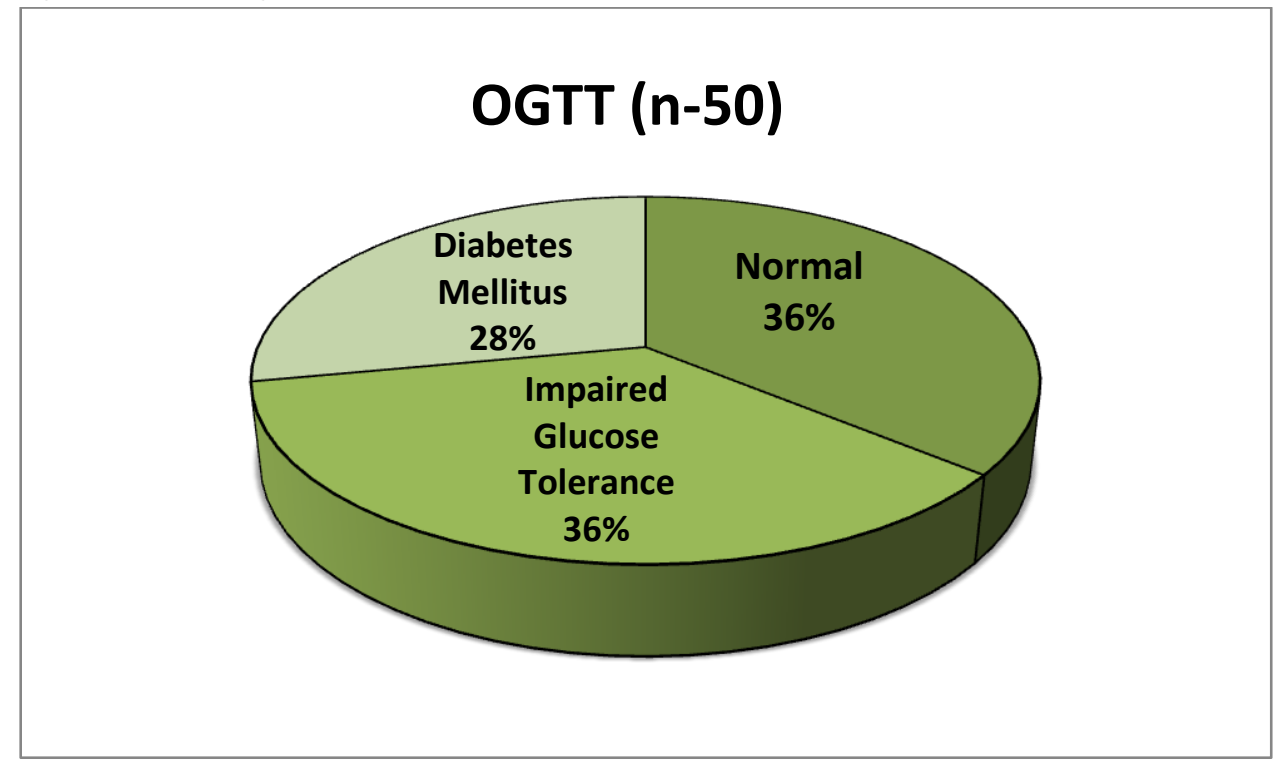

The mean of the oral glucose tolerance test performed in all the 50 patients were as follows: fasting- $112.1,1 / 2 \mathrm{hr}-142.8,1 \mathrm{hr}-175.2,1 \frac{1}{2} \mathrm{hrs}-$ 169.7 and $2 \mathrm{hrs}-162.5 \mathrm{mg} / \mathrm{dl}$. The results of the OGTT in these 50 patients were as follows: $18(36 \%)$ of them had normal results, $18(36 \%)$ of them had impaired glucose tolerance and the remaining 14 (28\%) of them had diabetes mellitus. other studies like the Naik B K, Mishra et al, Anklesari et al and Sarkar, the most common symptom was abdominal distension. Jaundice was present in $36 \%$ of the patients in this study, which was comparable with that done by Anklesari, where the $\%$ was also 36 . In our patients, fever was a manifestation in $28 \%$ of them which was comparable with Naik B K study.

\section{Discussion}

The commonest symptom in our study was anorexia, which was around $80 \%$. In this study the $\%$ of abdomen distension was 60 , which was the second commonest symptom. However in all the 
Table No 6 - Comparing OGTT results with previous studies

\begin{tabular}{|c|c|c|c|c|c|}
\hline OGTT & $\begin{array}{c}\text { Present study } \\
(\%)\end{array}$ & $\begin{array}{c}\text { Nishida T et } \\
\mathrm{al}^{17}(\%)\end{array}$ & $\begin{array}{c}\text { Hagel S }^{15} \\
(\%)\end{array}$ & $\begin{array}{c}\text { Müller MJ et } \\
\mathrm{al}^{18}(\%)\end{array}$ & $\begin{array}{c}\text { Hagel S }^{16} \\
(\%)\end{array}$ \\
\hline Diabetes & 28 & 21 & 34 & 37 & - \\
\hline IGT & 36 & 23 & 37 & 36 & 37 \\
\hline Normal & 36 & 39 & 29 & 27 & 33 \\
\hline
\end{tabular}

The results of the oral glucose tolerance test in this study,showed glucose metabolism disorders ie impaired glucose tolerance and diabetes in $64 \%$ of the cases.The number of patients with normal OGTT were 18 (36\%); 18(36\%) of them had impaired glucose tolerance and the remaining 14 (28\%) of them had diabetes mellitus.

This can be compared to the study carried out by Nishida $\mathrm{T}$ et al where the results of OGTT were as follows: 22 (39\%) of them were normal, 13(23\%) of them had IGT and the remaining $21(38 \%)$ had diabetes mellitus. This study also proved that diabetes mellitus and serum albumin were important independent negative predictors of survival. This also correlated with the study carried out by Hagel $\mathrm{S}$ which showed normal values in 23(29\%),IGT in $29(37 \%)$ and DM $27(34 \%)$. It was also proved beyond doubt that independent risk factors that lead to the development of carcinoma was diabetes mellitus along with older age and advanced liver fibrosis.

\section{Conclusion}

Cirrhosis liver is a very common disease in our country, with some of the major risk factors being alcohol, viral infection, carcinomas etc. The prognosis of chronic liver disease is varied and depends on a large number of parameters, including the stage of diagnosis of the disease. It is usually believed that chronic liver disease is associated with low blood sugar levels. However hypoglycaemia is most common in fulminant hepatic failure. Chronic liver disease, in contrast, presents with a significant percentage of glucose metabolism disorder (Impaired Glucose Tolerance/Diabetes Mellitus), and this is more evident with the later stages of the disease. The diabetes that occurs secondary to cirrhosis liver is given the term 'Hepatogenous Diabetes'. The degree of hyperglycaemia is an important prognostic factor in assessing the long term survival; and hence early detection by comprehensive diagnostic approach is mandatory. In normal individuals $80 \%$ of glucose is contributed by glycogenolysis after a prolonged fasting state. The hepatic glycogen is decreased in patients with chronic liver disease, which affects glycogenolysis adversely. Amino acids and lipids, the precursor of gluconeogenesis although are utilised prematurely to maintain the blood glucose levels, the fasting low blood sugar levels are not corrected. This is one reason for the utilisation of the oral glucose tolerance test (OGTT) in screening for diabetes in CLD, over fasting plasma glucose. The main mechanism for the carbohydrate intolerance in CLD includes insulin resistance and impaired secretion of insulin from the pancreatic cells.

The results in our study also showed significant percentage of patients with glucose metabolism disorders and this correlated well with the severity of the disease. That is, as the disease progressed (assessed by the Child Turcotte Pugh Scoring System) the degree of hyperglycaemia also worsened. The association of Diabetes Mellitus and cirrhosis liver leads to a more aggressive disease,with regard to the morbidity and mortality of the patient. The diagnosis of diabetes has hence very significant prognostic implications in the course of the disease.

These individuals are usually asymptomatic for diabetes till the end stage of the liver disease as true with any other diabetic patient. Hence screening of patients diagnosed with liver disease is of utmost importance at the earlier stages itself. This enables for the quicker and effective treatment of diabetes in these patients. But all said and done, there are few restrictions and 
contraindications for the treatment of diabetes in cirrhosis. However, with the recent development in the various therapeutic option, the outlook of the life of the patients has improved.

In conclusion, screening for diabetes with either fasting plasma glucose /OGTT should be done at the onset of the diagnosis of liver disease and at regular intervals; this is to enable the early diagnosis and efficient management of impaired glucose regulation in cirrhotics, and to improve the quality of life of these patients.
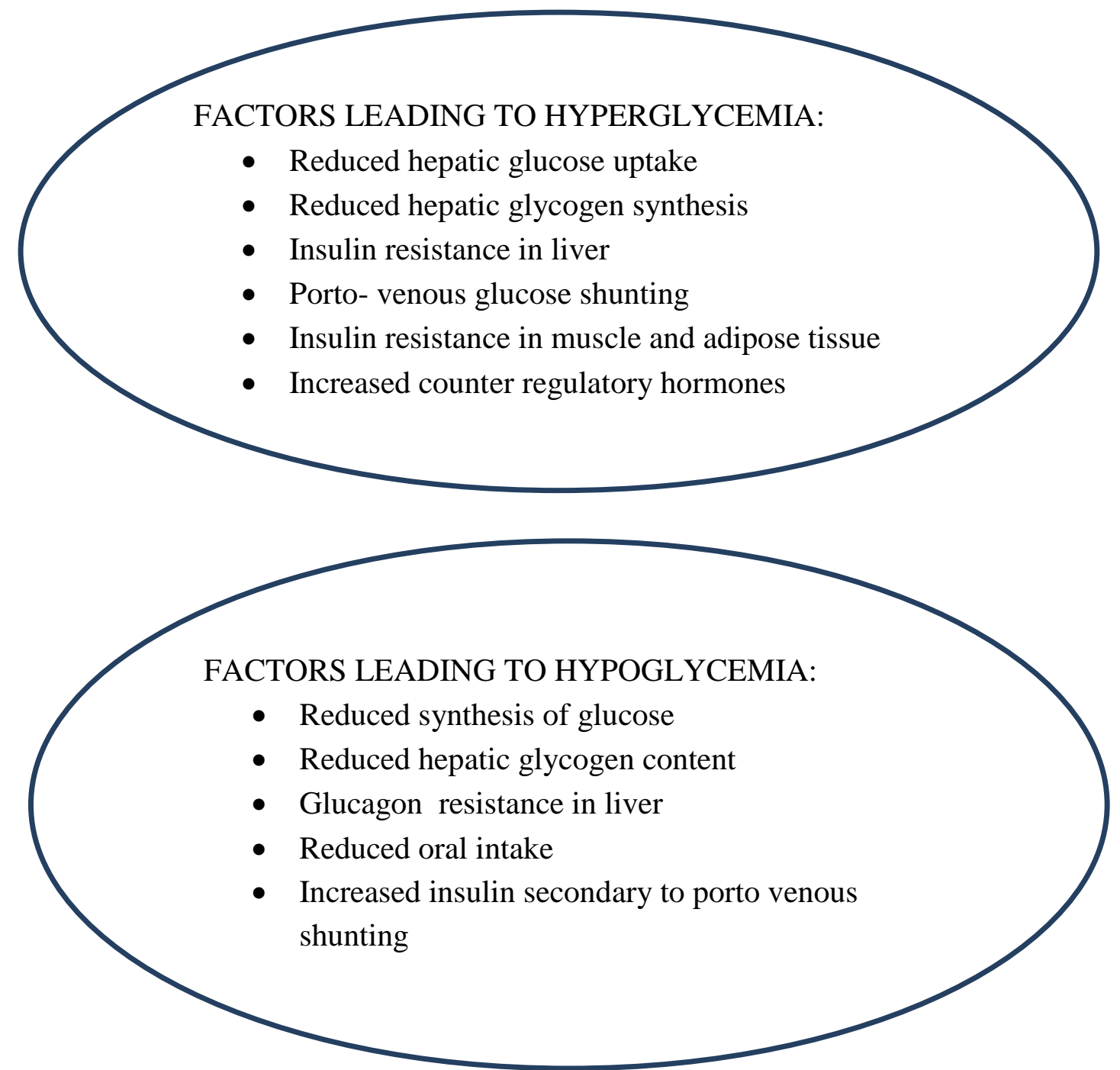

\section{Bibliography}

1. Kruzyska Y.T. carbohydrate metabolism: Textbook of Clinical Hepatology. Oxford University Press. $2^{\text {nd }}$ ed.1999; 257.

2. Daniel K.Podolsky and Kint J. Isselbacher: Derangements of Hepatic Metabolism Fauci, Brounwald, Isselbacker, Wilson ,Martin, Kasper,eds; Harrison's principles of Internal Medicine, Mc Graw Hill,14 th ed:1998.
3. Schneeweiss B, Grossinger W and Ference Petal: Energy Metabolism in patients with Acute and Chronic liver Disease. Hepatology, 1990 ; 11:387.

4. Manson A L, Law J.Y.N and Hoang $\mathrm{N}$ :Association of diabetes mellitus and chronic hepatitis $\mathrm{C}$ virus infection .Hepatology, 1999:29:3-28.

5. Felig P, Brown V, Robert A, Glucose homeostasis in viral hepatitis. New 
England Journal of Medicine, 1970: 283:26:1436.

6. Kruzyska Y.T, Glucose control in liver disease. Current Med.Lit.Gastroenterology 1992;11:9.

7. Majumdar G,Base $\mathbf{J}$ and Neelakantan, Diabetic state in chronic liver diseases. Journal of Associations of Physicians 24;6:359-366.

8. Lund,Schmidt and Decker T,Study of portal and cubital serum Insulin during oral,Portal and Cubital GTT.Acta.Med Scond,1975;197:275-281.

9. Naik B K and Narasmha R, Clinical Aspect of cirrhosis of Liver; J.A.P.I;11,10:791-793,1963.

10. Sarkar N,Pal J,Dasguptha,Agarwal $\mathrm{V}$, Kyjarwal D,etal :Clinical and Laboratory profile if chronic liver disease ,Paper presentation -88, APICON, API;51;12:2003.

11. Mendenhall CL: Alcoholic hepatitis, Clinical gastroenterology, 10;420,1981.

12. Brunt PW, Kew MC and Schener JJ studies on Alcoholic Liver Disease in Britain; Clinical and Pathological patterns related to a natural history. Gut; 1974;15:52-58.

13. Anklesari, glucose intolerance in Chronic Liver Disease; paper presention in APICON;1974

14. Mishra S.S \& Mishra R.N, Cirrhosis of Liver incidence, etiology \& Natural history in A.P.I;11,10:747-755,1963.

15. Hagel S, Bruns T, Schmidt C, Stallmach A. Impaired Oral Glucose tolerance test \& Diabetes Mellitus predict hort term prognosis of patient with Liver cirrhosis. Z gastroenterol 2009;47-P5_09. DOI: 10.1055/s-0029-1191971.
16. Hagel S, Bruns T, Hermann A, Schmidt C, Stallmach A. Abnormal glucose intolerance: a predictor of 30-day mortality in patients with decompensated Liver cirrhosis. Z Gasteroenterol. 2011 Mar;49 (3): 331-4. DOI:10.1055/s-0029-1245933

17. Nishida T, Tsuji S, Tsujii M, Amimitsu S et al . Oral glucose tolerance test predicts prognosis of patients with liver cirrhosis. Am J Gastroenterol. 2006 -Jan;(1):70-5

18. Muller MJ, Pirlich M, Balks HJ, Selberg O. Glucose intolerance in liver cirrhosis: role of hepatic and non-hepatic influences. Eur J Clin Chem Clin Biochem. 1994 Oct;32(10):749-58.

19. Shankar TP, Solomon SS, Duckworth WC, Himmelstein S, Gray S, Jerkins T, Bobal MA, Iyer RS. Studies of glucose intolerance in cirrhosis of liver. J Lab Clin Med. 1983 Oct;102(4):459-69.

20. Elichi Imano, Tsutomu Nishida, Michihiko Shibata, Tsutomu Kanda. Significance of oralglucose tolerance test for the diagnosis of diabetes mellitus in patients with liver cirrhosis. 1999 Nov;38(11):198. 